\title{
Modelling of a two-phase zone during condensation of R1234yf refrigerant in tubular minichannels under periodic dynamic disturbances
}

\author{
Waldemar Kuczyński ${ }^{1, *}$, and Aleksander Denis ${ }^{2}$ \\ ${ }^{1}$ Technical University of Koszalin, Department of Energy, av. Raclawicka 15-17, PL 75-620 Koszalin, Poland
}

\begin{abstract}
The paper presents an experimental analysis of the influence of dynamic disturbances on the size of the condensation zone of the appropriate new R1234yf environment-friendly refrigerant in circular minichannels. Experimental tests were conducted for single minichanells placed horizontally, with an inner diameter $d=\{1.44 ; 2.30 ; 3.30\} \mathrm{mm}$. A range of frequencies from $\mathrm{f}=0.2-5 \mathrm{~Hz}$ of periodically generated disturbances showed an explicit and unfavourable influence on the decrease of the length of the proper condensation region. This influence reduced the efficiency of the process. A computational model was developed to calculate the length of the two-phase region under disturbance conditions. The model proposed was verified by the results of the experiment, and compliance was obtained in the range of $\pm 25 \%$.
\end{abstract}

\section{Introduction}

A heat exchanger known as the condenser is one of the basic components of a power system that performs a reversed thermodynamic cycle. This component is crucial in terms of the overall efficiency of refrigeration systems or heat pumps. The basic input criteria for optimisation of the operation of such systems is the available heat transfer area of the condenser. This applies both to the heat rejection by the working fluid (main medium) and heat absorption by the coolant (auxiliary medium). The hydraulic losses also play an essential role in the process. Both heat transfer processes (heat rejection and heat absorption by the working fluid) affect the heat transfer rate in a different way. Therefore, it is very important to provide proper conditions that intensify the overall heat transfer coefficient of the condenser [1 - 7]. Modern trends in the design of power machinery are focused on the energy efficiency. As a consequence, the power systems are becoming smaller and smaller owing to the employment of mini and microchannels as well as new pro-ecological refrigerants [8]. According to the 2020 forecast, the share of miniaturized devices should reach up to $25 \%$ of all refrigeration, power, medical, IT, aviation and aerospace equipment produced [8].

The manufacturing process of the above-mentioned systems require the employment of heat exchangers (condensers and evaporators) based on channels with a hydraulic diameter significantly smaller than the socalled conventional ones. The classification of those systems has already been developed. This classification is based on the size of the internal diameter of the channel -channels with internal diameter below the range $3-6 \mathrm{~mm}$ are known as minichannels [9].

Such systems, as well as those conventional ones, require properly selected working fluid. So far, one of the most commonly used refrigerants were the so-called fluorinated greenhouse gases, especially: hydrofluorohydrocarbons, perfluorohydrocarbons, sulphur hexafluoride and other fluids that contain fluorine. The fluorinated greenhouse gases group includes the R134a refrigerant. Although this refrigerant is commonly used in currently operated systems, it has been phased-out since 01.01.2017 and the process should be completed by 31.12.2020. The scope of the applications of this refrigerant covers air conditioning systems in mobile applications and heat pumps. The suggested substitute for the R134a refrigerant is the chemical compound labelled R1234yf. Its GWP index (Global Warming Potential) is very small, just 4, comparing to 1430 in case of its predecessor R134a $[10,11]$.

During the condensation phase change in minichannel based systems, as well as in conventional systems, there are plenty of thermal-hydraulic, structural and operational problems. Those problems include the possibility of the occurrence of unsteady working conditions induced by instabilities of internal (static) and dynamic (external) origin. This paper pays special attention on phenomena connected with the onset and propagation of disturbances in the systems that perform condensation phase change of refrigerants [12 - 16].

The static instabilities are inherently connected with the change of the state parameters of the system. Basing on the record of state parameters evolution in time, it is possible to predict when the static instability will occur

Corresponding author: waldemar.kuczynski@tu.koszalin.pl 
(the system is considered deterministic). As a result, the system will reach an another steady state or will oscillate around this state. A perfect example of static instabilities is the flow excursion (also known as the Ledinegg instability) [17,18]. It involves pressure drop due to hydraulic losses during phase change. This instability may occur in a specific state of the system when the total pressure drop decreases due to increase of flow rate.

When the system is under influence of thermal or hydraulic forces that introduce inertia, the flow becomes unstable and the dynamic instabilities occur. The exact state of the system becomes probabilistic or stochastic. The flow instabilities in two-phase media (liquid-gas) propagate in a form of two phenomena: a pressure wave (acoustic wave travelling with the speed of sound), flow rate fluctuations (due to the oscillations of void fraction) and temperature instability also known as the evaporation front (for the evaporation phase change) or condensation front (for the condensation phase change) [12-16, 19-23].

Those phenomena have a wave character described with different propagation velocities. The acoustic waves are characterized by high frequency, while flow rate and temperature oscillations are characterized by much smaller frequency.

Previous experimental research on influence of instabilities on flow phase change of refrigerants in minichannels allowed partial recognition of those phenomena $[1-6,12-16]$. However, there are no publications that concern those phenomena regarding new pro-ecological refrigerants that are meant as a substitutes for those being phased out.

\section{Computational model}

During the condensation process in tubular minichannels and conventional condensers of compressor based refrigeration systems, it is possible to distinguish three characteristic sub-zones inside the condenser: precooling of superheat vapour, proper condensation (twophase) and sub-cooling of the liquid. Figure 1 shows the temperature distribution of the refrigerant and the coolant along the condenser, including the abovementioned sub-zones.

In the first sub-zone, the superheated vapour is being cooled-down until it reaches the condensation temperature (saturated steam with quality $\mathrm{x}=1.0$ ). From this point on, provided that there is a temperature gradient at the wall, the two-phase proper condensation sub-zone begins. The flow condensation process is determined by process parameters and takes place until the saturated liquid state $(x=0.0)$ is reached. From this point on, the third sub-zone begins. It is responsible for the cool-down of the liquid. The most intense heat transfer mode is the two-phase flow condensation that takes place inside the proper condensation sub-zone. Single phase cool-down and sub-cooling sub-zones are characterized by much lower heat transfer coefficients. The onset of instabilities, both static and dynamic, may cause the disturbance of distribution of sub-zones inside the condenser, including a shrinkage of the active area of proper condensation characterized by the highest heat transfer coefficient [12].

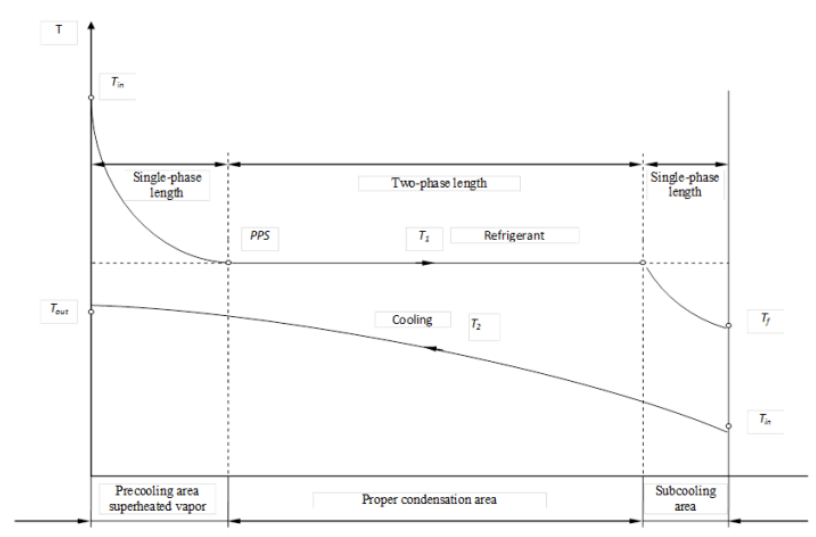

Fig. 1. Diagram of the distribution of the temperature of refrigerant and cooling medium during condensation inside the horizontal pipe of condenser; $T_{p}-$ superheated vapour temperature, $T_{s}$ - temperature of condensation (saturation), $\mathrm{T}_{\mathrm{f}}-$ final temperature of the process, $T_{i n}$., $T_{\text {out }}$. - temperature of cooling medium, at the beginning and end of process respectively, PPS - starting point of condensation [12].

The author of the paper [7] suggested a theoretical model that allows one to determine the size of each of the sub-zones of the condenser under steady-state operation. However, in order to determine the size of the proper condensation sub-zone for the R1234yf refrigerant for flow condensation inside minichannels under periodic dynamic instabilities, a novel model developed for the R134a and R404A refrigerants was used [12].

\subsection{Apparatus}

The experimental research is aimed at a verification the presented mathematical model. At the same time, it allowed the Authors to determine the influence of periodically generated hydrodynamic disturbances on the size of each of the heat transfer sub-zones during flow condensation of the R1234yf refrigerant inside tubular minichannels.

During experimental research, the tubular minichannels made of stainless steel with internal diameters $\mathrm{d}=\{1.44 ; 2.30 ; 3.30 \mathrm{~mm}\}$ were used. The research was conducted using an apparatus that was specially designed for this experiment. The same apparatus was previously used for the investigation of the R134a refrigerant. Minor changes were introduced in order to adjust the installation to the new pro-ecological refrigerant R1234yf. The Diagramdiagram of the apparatus in presented in Figure 2. Figure 3 shows the test section in details.

The instrumentation of the apparatus allowed the Authors to measure the following parameters:

- pressure $p_{s}$ of the refrigerant during flow along the tubular minichannel,

- wall temperature along the tubular minichannel,

- pressure of the refrigerants at the inlet and outlet of the test section $p_{\text {out }}, p_{\text {in }}$, 
- temperature of the refrigerant at the inlet and outlet of the test section $T_{w e j}, T_{w y j}$,

- mass flow rates of the refrigerant $\dot{m}_{R I 234 a}$ and the cooling water $\dot{m}_{\mathrm{H}_{2} \mathrm{O}}$.

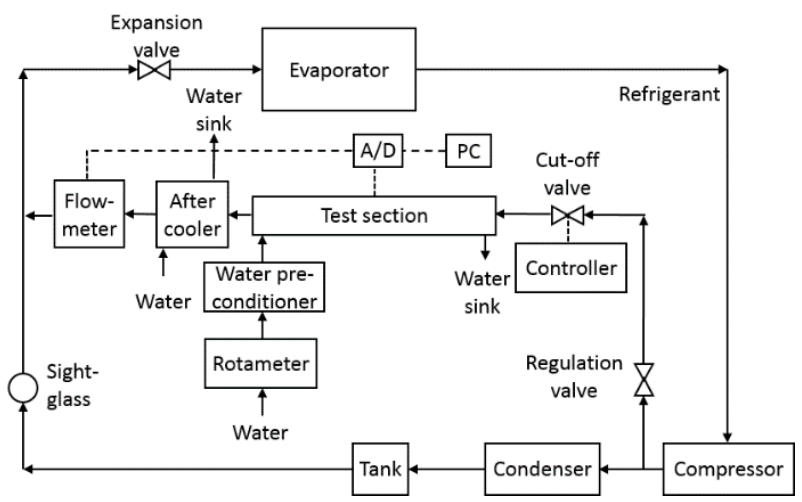

Fig. 2. Diagram of the apparatus.

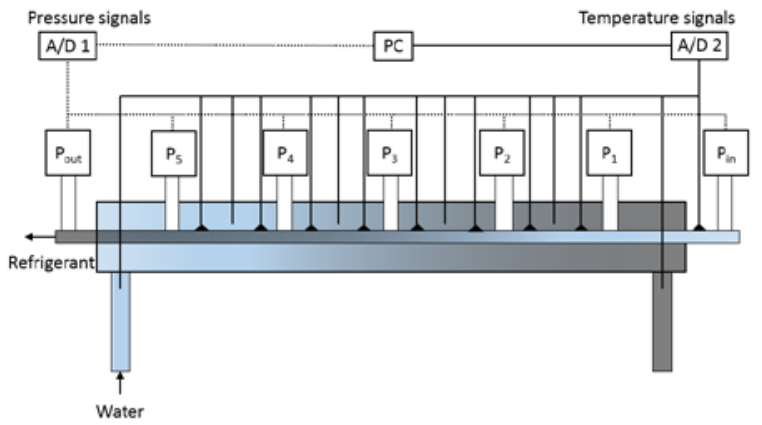

Fig. 3. Diagram of the test section.

The measurements were done for the following intervals of the parameters:

- mass flux of the refrigerant $G=100 \div 4100$ $\mathrm{kg} /\left(\mathrm{m}^{2} \cdot \mathrm{s}\right)$,

- $\quad$ saturation temperature of the refrigerant $T_{s}=20 \div$ $55^{\circ} \mathrm{C}$,

- frequency of the instabilities $f=0,20 \div 5 \mathrm{~Hz}$.

The research was done for a constant value of mass flow rate of the refrigerant during the steady state that preceded the onset of the instabilities. The test section with channel length $L=880 \mathrm{~mm}$ was armed with piezoelectric pressure sensors and K-type thermocouples maintaining a constant distance between the sensors. The voltage based signals collected from the pressure and temperature sensors, and the flow meter were measured using:

- thermoelectric temperature sensors made of $\mathrm{NiCr}-\mathrm{Ni}$ (each sensor was individually calibrated prior to the research) coupled with A/D adapter USB PersonalDaq/3000 with $P D Q 30$ extension module,

- piezoresistive pressure sensor (Cerabar TPMP131 type) coupled with $\mathrm{A} / \mathrm{D}$ adapter,

- flow meter Coriolisa Promass 80A.

All the equipment was connected to a PC with data acquisition and processing software.

\subsection{Mathematical model}

According to the temperature distribution of the refrigerant flowing along the horizontal minichannel, shown in Figure 1, during the condensation of the R1234yf refrigerant, it is clearly visible that the proper condensation sub-zone onsets for both steady and unsteady conditions. If at the inlet that supplies superheated vapour $T_{p}$ to the minichannel, there is an electromagnetic regulation valve, then it is possible to generate periodic hydrodynamic instabilities simply by alternating opening and closure of this valve. The frequency of those instabilities can be calculated using the following formula [24]:

$$
f=\frac{1}{\tau_{o}+\tau_{z}}
$$

where: $\tau_{o}$ is the opening time and a $\tau_{c}$ is the closure time of the valve that supplies the refrigerant to the test section.

The possibility of determining the length of the twophase sub-zone $L$ for the R1234yf under previously stated conditions was also taken into account. During the modelling process, the following assumptions were made:

- inside the straight, axisymmetric tubular minichannel with the length $L$ and constant internal diameter $\mathrm{d}$, there is a mass flow rate of refrigerant $\dot{\mathrm{m}}$;

- the refrigerant condenses during the flow along the tubular minichannel until it reaches the saturated liquid state (quality $\mathrm{x}=0.0$ ) and then it is being sub-cooled;

- periodic instabilities with constant frequency are generated by the opening and closure of the cut-valve at the inlet of the refrigerant to the tubular minichannel;

- the mass flow rate $\dot{m}$ of the refrigerant is under oscillatory fluctuations;

- the instabilities propagate along the axis of the horizontal channel of the length $\mathrm{L}$ in a form of pressure wave with velocity $v_{p}$ that is a functions of the amplitude and frequency.

From the condensation heat balance for elementary length of tubular minichannel in the two-phase sub-zone, it results:

$$
d \dot{Q}_{i}=q_{i} \cdot \pi \cdot D \cdot d L_{T F P},
$$

where:

$d \quad$ - internal diameter of the minichannel,

$q_{i} \quad$ - local heat flux,

$d L_{T F P}$ - elementary length of the minichannel.

The heat flux removed along within the two-phase subzone of the minichannel is described by the following formula:

$$
\dot{Q}=\dot{m} \cdot\left(h_{G}-h_{L}\right)=\dot{m} \cdot r,
$$

where:

$\dot{m} \quad$ - average mass flow rate under steady conditions,

$h_{G} \quad-$ specific enthalpy of the refrigerant at the inlet to the two-phase sub-zone (saturated vapour with quality $\mathrm{x}=1$ ), 
$h_{L} \quad-\quad$ specific enthalpy of the refrigerant at the outlet of the two-phase sub-zone (saturated liquid with quality $\mathrm{x}=0$ ).

Combining Equations (2) and (3), it can be written as follow:

$$
d \dot{Q}=\dot{m} \cdot d h
$$

By introducing a constant $\mathrm{C}=\pi \cdot \mathrm{D}$ into Equation (2), Equation (4) can be rewritten:

$$
C \cdot q_{i} \cdot d L_{T F P}=\dot{m} \cdot d h
$$

The heat flux qi from Equation (5) should be regarded as a local parameter that depends both on time and the distance from the channel's inlet up to the beginning of the two-phase sub-zone: $q_{i}=q_{i}(L, t), d_{h}$ however is stands for the local elementary drop of the specific enthalpy in this cross-section.

The change (oscillations) of the mass flow rate of refrigerant caused by the periodically generated hydrodynamic instabilities can be treated as a propagation of a small amplitude and frequency monochromatic wave along the axis of the horizontal channel. Based on the following studies [25,26], the oscillatory variation of the mass flow rate at the minichannel's inlet can be determined using the following formula:

$$
\dot{\mathrm{m}}_{\text {inst. }}=\dot{\mathrm{m}} \cdot[1+\mathrm{A} \cdot \sin (2 \cdot \pi \cdot f \cdot \tau)
$$

where $\dot{m}, A, f$ and $\tau$ are mass flow rate before the onset of oscillations, amplitude of the oscillations, frequency and time, respectively. The maximum amplitude of the oscillations of mass flow rate $A$ equals to the undisturbed radius of internal surface of the condensate film. This amplitude can be calculated using the following formula:

$$
A=A_{0} \cdot \exp ^{\omega \tau k}
$$

The minimal thickness of the undisturbed condensate film equals to: $\delta=\frac{r_{0}}{2}$, where $r_{0}$ is the internal diameter of the minichannel $[12,19]$. The $A_{0}$ parameter stands for the initial amplitude of the instabilities. Its value depends on the vapour inlet pressure and equals $A_{0}=-p_{G}$. The $\omega$ parameter stands for an increase of the amplitude $A$, and $k$ stands for the wave number [19]:

$$
k=\frac{2 \pi}{\lambda}=\frac{\omega}{V_{p}},
$$

where:

$\lambda-$ wave length,

$v_{p}$ - propagation velocity of the instability, $v_{p}=\frac{l}{\Delta \tau}$,

$l$ - distance between the pressure sensors,

$\Delta \tau-$ delay of the pressure signal, $\omega-$ pulsation (angular frequency) of the base parameter, $\omega=\frac{2 \pi}{T}[21,23]$,

$T \quad$ - wave period $\left(T=t=t_{o}+t_{c}\right)$.

By combing Equations (5) and (6), the following is obtained:

$$
\dot{\mathrm{m}}_{\text {inst. }} \cdot \mathrm{dh}=\mathrm{C} \cdot \mathrm{q}_{\mathrm{i}} \cdot \mathrm{dL} \text { TFP, }
$$

and after integration:

$$
\int_{h_{1}}^{h_{2}} \dot{m}_{i n s t .} \cdot d h=\int_{0}^{L} C \cdot q \cdot d L_{T F P},
$$

and further:

$$
\dot{m}_{\text {nies. }} \cdot \Delta h=C \cdot \int_{0}^{L} q \cdot d L_{T F P} .
$$

Equation (11) was employed to determine the length of the proper condensation sub-zone of the tubular minichannel. It can be solved using numerical methods, provided that the distribution of heat flux $q_{i}=q_{i}(L, t)$ is known. Assuming that the length of the two-phase subzone depends on the frequency of periodically generated instabilities $f$, the problem is simplified.

Calculations of the average heat flux $\bar{q}$ along the length of the proper condensation sub-zone $L_{T F P}$ inside the minichannel were made following the method presented in [7]. According to the authors of this paper, the average heat flux $\bar{q}$ along the length of the proper condensation sub-zone $L$ equals to:

$$
\bar{q}=\frac{1}{L} \int_{0}^{L} q(L) d L_{T F P} \text { thus } \int_{0}^{L} q(L) d L=\bar{q} \cdot L_{T F P}
$$

and finally:

$$
\dot{m}_{\text {inst... }} \cdot \Delta h=C \cdot \bar{q} \cdot L_{T F P},
$$

where:

$$
\dot{\mathrm{m}}_{\text {inst. }}=\left(\mathrm{G}_{\text {inst. }} \cdot \mathrm{F}\right) \text {. }
$$

Assuming that the internal cross-section of the channel is constant $d A / d z=0$, the length of the two-phase proper condensation sub-zone $L_{T P F}$ can be calculated using the following formula:

$$
L_{\text {TPF }}=F \cdot \frac{G_{\text {inst. }} \cdot \Delta h}{C \cdot \bar{q}}
$$

For the minichannel with a constant internal crosssection area $F=\frac{\pi \cdot d^{2}}{4}$ and the perimeter $C=\pi \cdot d$, Equation (15) takes the following form:

$$
L=d \cdot \frac{G_{\text {inst. }} \cdot \Delta h}{4 \cdot \bar{q}},
$$




$$
L=d \cdot \frac{G_{\text {inst }} \cdot r \cdot(1-x)}{4 \cdot \bar{q}}
$$

where $r$ is the latent heat of condensation, and $\bar{x}$ is the average quality of the refrigerant.

Based on Formula (17), the length of the two-phase condensation sub-zone can be calculated, provided that all the assumptions of the model are satisfied.

Taking into account the fact that the external instabilities are induced by the valve, the model included the valve's characteristics - relative valve flow coefficient $k_{v}$. This coefficient determines the relation between the valve position and the flow rate. Depending on pressure drop at the valve, there are two main characteristics: internal and operational. The value of $k_{v}$ is determined as follow [27]:

$$
k_{V}=\frac{K_{V}}{K_{\text {1 } 00}},
$$

where:

$K_{v} \quad-$ computational valve flow coefficient according to [27] - this is the characteristic factory size of the valve, $\mathrm{m}^{3} / \mathrm{h}$,

$K_{v 100}-$ nominal (catalogue) value of valve flow coefficient according to the manufacturer, $\mathrm{m}^{3} / \mathrm{h}[27]$.

The coefficient $K_{v w}$ is presented, according to [27], in the following form:

$$
K_{V W}=10 \cdot \dot{m} \cdot \sqrt{\frac{V_{2}}{\Delta p}},
$$

Provided that: $p_{2} \geq \frac{p_{1}}{2}$,

where:

$\dot{m}$ - mass flow rate of the refrigerant,

$\Delta p$ - pressure drop at the valve,

$v_{2} \quad$ - specific volume of vapour for $p_{2}$ and $T_{1}$,

$p_{1} \quad$ - pressure at the inlet,

$p_{2} \quad$ - pressure at the outlet,

$T_{l}$ - temperature of the refrigerant before the valve,

Equation (17), after substitution of Equation (18), takes the following form:

$$
L=k_{v} \cdot \frac{d \cdot G_{n s t .} \cdot r \cdot(1-\bar{x})}{4 \cdot \bar{q}} .
$$

\section{Experimental results}

The dynamic external instabilities during condensation of the R1234yf refrigerant inside tubular minichannel manifested themselves in the form of fluctuations of the mass flow rate and, consequently, the fluctuations of the pressure and $p_{s}$ and the saturation temperature $T_{s}$. Figures $4-6$ show the examples of oscillations of the mass flow rate, pressure $p_{s}$ and wall temperature $T_{\text {wall }}$.

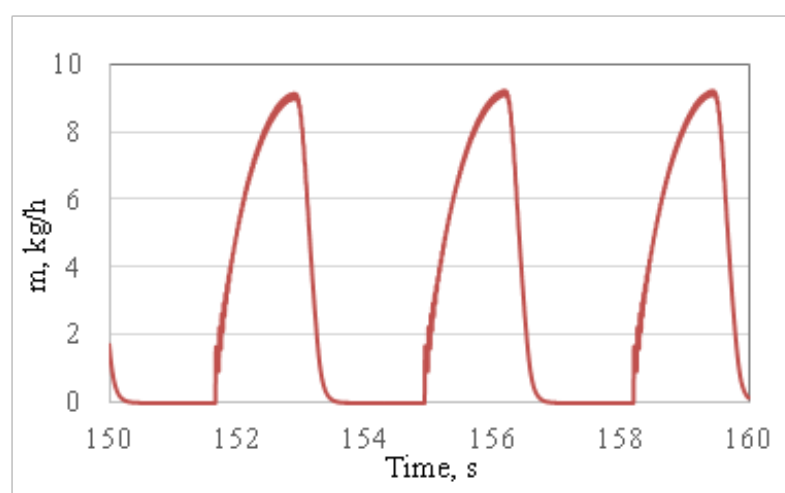

Fig. 4. Sample of flow oscillations.

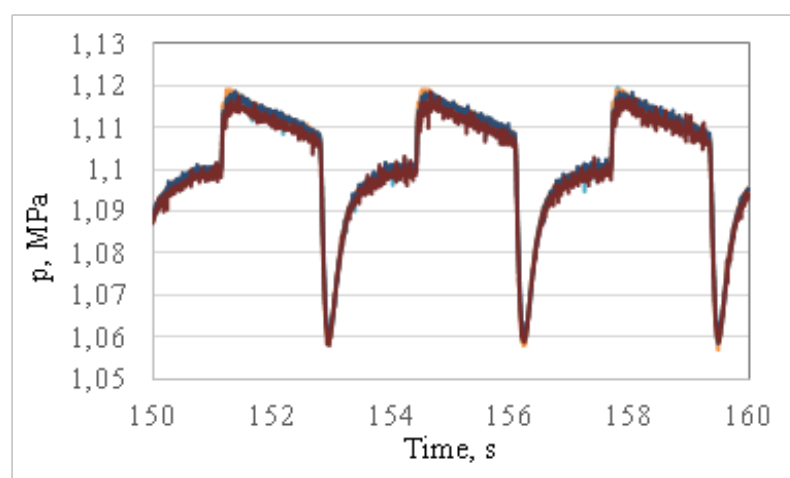

Fig. 5. Sample of pressure oscillations for multiple sensors.

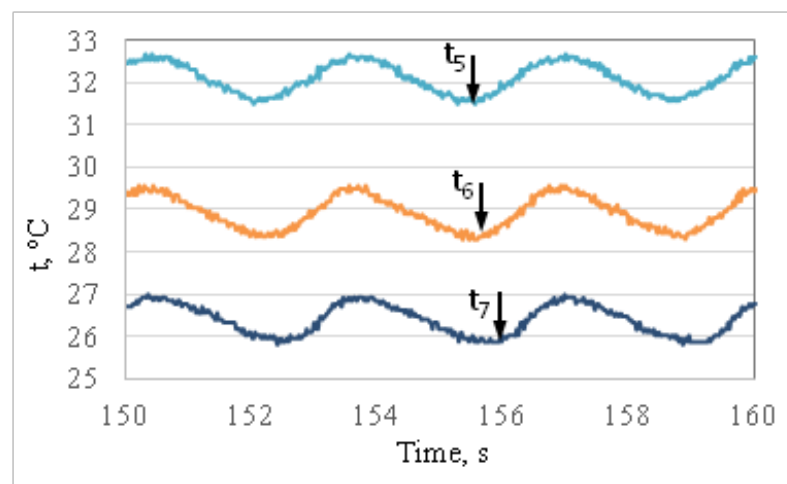

Fig. 6. Sample of temperature oscillations for multiple sensors.

Based on the obtained experimental results, the temperature distribution of the refrigerants along the investigated minichannel was obtained. Figure 7 shows a summary that includes the distribution of sub-zones depending on the frequency of the periodic instabilities for minichannel with internal diameter $\mathrm{d}=1.44 \mathrm{~mm}$.

The summary presented in Figure 7 allows one to visualize the influence of hydrodynamic instabilities on condensation inside the tubular minichannels. The external instabilities were generated through a periodic sudden closure of the inflowing refrigerant. As a result, the length of both superheat removal and proper condensation zones contracted, while the sub-cooling section expanded. The higher the closure time is, the smaller superheat removal and proper condensation zones are. The change in sub-zones distribution is significant, thus the thermal performance of the condenser drops. An increase of the valve closure time 
translates into a lower frequency of the instabilities $f$, see Equation (1).

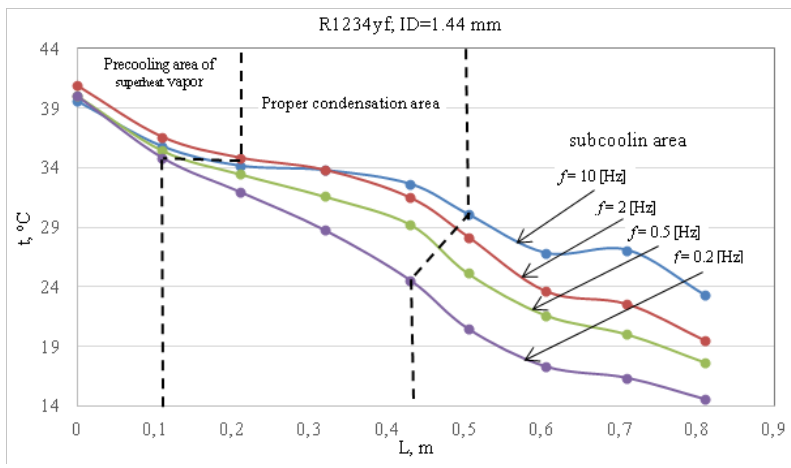

Fig. 7. Results of the experimental investigations concerning the influence of periodically generated instabilities on the length of the of the superheat removal, proper condensation and sub-cooling sub-zones for the R1234yf refrigerant depending of the frequency $f$ of the generated disturbances in mini-channels with internal diameter $\mathrm{d}=1.44 \mathrm{~mm}$.

\section{Computational results}

The computational results of the length of the two-phase condensation sub-zone $L_{c a l}$. were compared with the experimental results for the R1234yf refrigerant for minichannels with internal diameters $d=\{1.44 ; 2.30$; $3.30\} \mathrm{mm}$.

Within the scope of the statistical analysis that aimed to compare numerical results obtained using the presented model and the experimental results, the relative mean absolute error (RMAE) criterion was used [24]:

$$
R M A E=\frac{1}{n} \cdot \sum_{i=1}^{n} \frac{\left(y_{i}-y_{i}\right)}{y_{i}} \cdot 100 \%,
$$

where:

$\hat{y}_{i}-$ simulated value (numerical value obtained using a verified model),

$y_{i}$ - empirical value (experimental),

$n$ - number of measurement points.

Based on the calculations, it was found that the relative mean absolute error in the case of the two-phase proper condensation sub-zone does not exceed $\pm 25 \%$. It was confirmed by comparing the experimental results with calculations using Formula (19). The results are shown in Figure 8. The value or error is higher than in the case of an estimation of the proper condensation sub-zone size for the $\mathrm{R} 134 \mathrm{a}$ refrigerant $( \pm 15 \%)$. This may be due to the properties of the R1234yf refrigerant and requires further investigations.

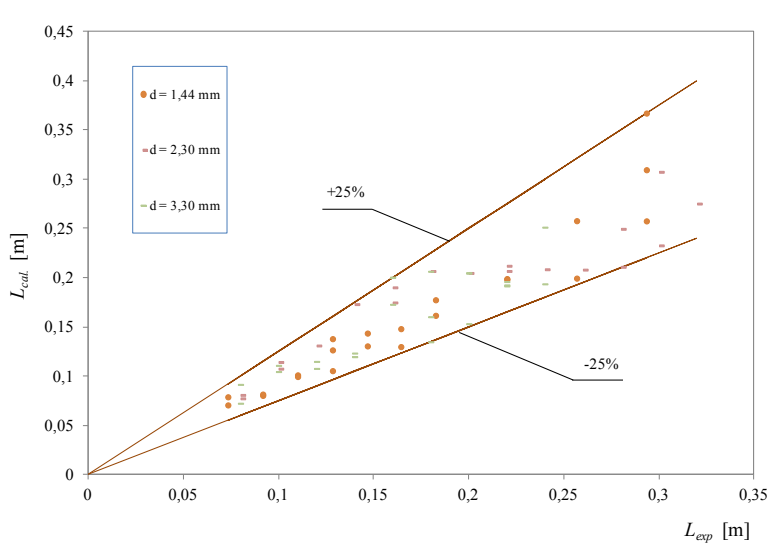

Fig. 8. Comparison of numerically obtained length of the twophase proper condensation sub-zone Lcal according to Formula (19) with experimental results $L_{\text {exp }}$.

\section{Conclusions}

1) Periodically generated external dynamic instabilities during condensation of the R1234yf refrigerant in minichannels with internal diameter $\mathrm{d}=$ 1.44; 2.30 and $3.30 \mathrm{~mm}$, just like in the case R134a refrigerant, cause fluctuations of the mass flow rate of the working fluid that leads to further wave changes of the pressure and wall temperature.

2) Periodically generated dynamic instabilities within the frequency range $\mathrm{f}=0,25-5 \mathrm{~Hz}$ have an influence on the size of the sub-zones inside the minichannel based condenser, especially, the size of the proper condensation sub-zone. Due to the highest heat transfer coefficient, the size of this zone determines the thermal performance of the condenser. The frequency of the generated instabilities $f$ has a negative influence on the thermal performance of the condenser.

3) The model presented in this paper was compared with the experimental results. The difference between experimental and numerical results did not exceed $\pm 25 \%$ range. The value of this error is significantly higher than in the case of the estimation of the proper condensation sub-zone for the R134a refrigerant $( \pm 15 \%)$.

4) Nevertheless, despite the higher error of the model for the R1234yf refrigerant, Formula (19) is still applicable.

\section{References}

1. T. Bohdal, Bubble boiling in flow of refrigerating media. Journal of Mechanical and Energy Engineering, Vol. 1 No. 1 (41), June 2017, pp. 57 64.

2. T. Bohdal, M. Kruzel, M. Sikora, Analisys of heat transfer coefficient during refrigerant condensation in vertical pipe minichannel. Journal of Mechanical and Energy Engineering, Vol. 1 No. 1 (41), June 2017, pp. $65-70$.

3. R. Laskowski, M. Jaworski, Maximum entropy generation rate in a heat exchanger at constant inlet parameters. Journal of Mechanical and Energy 
Engineering, Vol. 1 No. 1 (41), June 2017, pp. 65 70.

4. M. Sikora, Flow structures during refrigerants condensation. Journal of Mechanical and Energy Engineering, Vol. 1 No. 1 (41), June 2017, pp. 101 106.

5. T. Bohdal, M. Kruzel, M. Sikora, An investigation of heat transfer coefficient during refrigerants condensation in vertical pipe microchannel. Journal of Mechanical and Energy Engineering, Vol. 1(41), No. 2, November 2017, pp. 163 - 170.

6. W. Kuczynski, A. Denis, Modeling the instabilities of the condensation process of the R134a and $R 404 A$ refrigerants in pipe minichannels in the conditions of periodic hydrodynamic disturbances. Journal of Mechanical and Energy Engineering, Vol. 1(41) No. 2, November 2017, pp. 179 - 188.

7. A. Paliwoda, Calculation of evaporators and condensers of refrigeration equipment. Vol. I Basic equations and dependencies. Vol. II. Length of pipes and flow resistance of refrigerants. Refrigeration Year IX. Notebook 3, 1974, pp. 1 - 6. in Polish

8. Z. Gnutek, A. Nemś, Trends in the development of machinery and power equipment in the era of miniaturization. Materials of the XXX Congress of Thermodynamics, Wroclaw 2008, volume I, 318 324. in Polish

9. S.M. Ghiaasiaan, Two-phase flow, boiling, and condensation in conventional and miniature systems. Cambridge University Press 2008, pp. 68 76.

10. D. Colorado, J.A. Hernandez, W. Rivera, Comparative study of a cascade cycle for simultaneous refrigeration and heating operating with ammonia, $\mathrm{R} 134 \mathrm{a}$, butane, propane and $\mathrm{CO}_{2}$ as working fluids. International Journal of Sustainable energy 2011, 06.

11. D.H. Kim, H.S. Park, M.S. Kim, Optimal temperature between high and low stage cycles for R134a/R410A cascade heat pump water heater system. Experimental Thermal and Fluid Sci 2013, vol. 47, s. 172-179.

12. W. Kuczynski, H. Charun, Modeling of a TwoPhase Region Length of the Condensation of R134a and R404A Refrigerants in Pipe Minichannels With Periodic Hydrodynamic Instabilities. Heat Transfer Engineering Volume 35, Issue 9, pp. 850-862.

13. W. Kuczynski, Modeling of the propagation of $a$ pressure wave during the condensation process of $R 134$ a refrigerant in a pipe minichannel under the periodic conditions of hydrodynamic disturbances. International Journal of Heat And Mass Transfer Volume 56, Issue 1-2, pp. 715 - 723.

14. W. Kuczynski, T. Bohdal, H. Charun, Impact of Periodically Generated Hydrodynamic Disturbances on the Condensation Efficiency of R134a Refrigerant in Pipe Mini-Channels.
Experimental Heat Transfer, Vol. 26, Issue 1, pp. 64 $-84$.

15. W. Kuczynski, Pressure wave propagation during the condensation process of the $R 404 \mathrm{~A}$ refrigerant in mini-condenser under periodic hydrodynamic disturbances. International Journal of Heat And Mass Transfer Volume 67, pp.404-415.

16. W. Kuczynski, H. Charun, T. Bohdal, Modeling of temperature instabilities during condensation of R134a refrigerant in pipe minichannels. International Journal of Heat And Mass Transfer. Volume 111, pp.83-93.

17. S. Kakac, B. Bon, Areview of two-phase flow dynamic instabilities in tube boiling systems. International Journal of Heat and Mass Transfer 51 (3-4), 2008, pp. $399-433$.

18. T. Zhang, T. Tong, J-Y. Chang, Y. Peles, R. Prasher M.K Jensen, J. Wenf, P. Phelan, Ledinegg instability in microchannels. International Journal of Heat and Mass Transfer 52, 2009, pp. 5661 - 5674.

19. K.H. Ardron, R.B. Duffey, Acoustic wave propagation in a flowing liquid-vapor mixture. International Journal Multiphase Flow 4, 1977, pp. $303-322$.

20. L. Cao, S., Kakaç, H.T. Liu, P.K. Sarma, The effects of thermal non-equilibrium and inlet temperature on two-phase flow pressure drop type instabilities in an upflow boiling system. Int. J. Therm. Sci. 39, 2000, pp. $886-895$.

21. O. Comakli, S. Karsli, M. Yilmaz, Experimental investigation of two phase flow instabilities in a horizonatl in-tube boiling system. Energy Conversion and Management 43, 2002, pp. 249 268.

22. Y. Ding, S. Kakaç, Dynamic instabilities of boiling two-phase flow in a single horizontal channel. Experimental Thermal and Fluid Science, 1995, pp. 327 - 342.

23. A.N. Pavlenko, V.V. Lel', Model of self-maintaining evaporation front for superheated liquids. Proc. 3th Int. Conference on Multiphase Flow, ICMF'98, Lyon, France 4, 1998, pp. 3-5.

24. P. Kartaschoff, Frequency and Time, Publishing House of Communications and Communications Warsaw 1995. in Polish

25. T.N. Veziroglu, Two-phase flow instabilities, final report. NSF Project CME 79-20018, Clean Energy Research Inst. Coral Gables, FL 1983.

26. G.B. Whitham, Linear and nonlinear waves. Wiley, New York 1974.

27. International Standard IEC 60534-1. 1987., 15 